\title{
Modeling Tsunami Run-Up and Draw-Down on the Beach
}

\author{
William Noland, North Central College (wpn5011@psu.edu) \\ Dylan Smith, University of Connecticut (dylan.m.smith@uconn.edu) \\ Seth Selken, Iowa State University, (sselken@iastate.edu) \\ Adviser: Dr. Sergei Fomin, California State University, Chico \\ (sfomin@csuchico.edu)
}

\begin{abstract}
Previous literature has investigated the run-up and draw-down of tsunami waves on a one-dimensional, constant-sloped beach, but the existing solutions are complex and computationally unwieldy. Our research aims to establish a simpler model while still obtaining accurate results. We do so by using a quasi-linear theory derived from the nonlinear shallow-water wave equations. These equations are considered over a linear beach with properly imposed initial and boundary conditions. The main difficulty in solving this problem is the moving boundary associated with the shoreline motion. To eliminate this difficulty, we apply an appropriate substitution to the spatial variable, and thus replace the moving boundary of the computational domain with a stationary boundary. A key feature of our tsunami problem is the presence of the small parameter $\varepsilon=\frac{\eta_{0}}{h_{0}}$, where $\eta_{0}$ is the characteristic amplitude of the wave and $h_{0}$ is the characteristic depth of the ocean. Due to the presence of this small parameter, the problem can be essentially linearized using the method of perturbations and then solved analytically via an integral transformation. Our explicit solution enables us to swiftly predict the behavior of the wave using an essentially linear model. We test the accuracy of our model against the numerical solution obtained using Mathematica, and find minimal discrepancies. Finally, we extend our results to a modified beach configuration that more accurately reflects real-world shoreline topography.
\end{abstract}

\section{Introduction}

\subsection{Background Information}

Tsunamis cause widespread and devastating damage to coastal communities. These natural disasters can quickly harm the lives of thousands due to their great power and inertia. Tsunamis are typically the result of underwater earthquakes or underwater volcanic eruptions which cause major displacements of water [2] [7]. Although they have minimal amplitude (usually no greater than $30 \mathrm{~cm}$ ), making them extremely difficult to spot offshore, tsunamis can have lengths of up to hundreds of kilometers, generally far greater than the depth of the ocean. As the tsunami reaches the shoreline the speed decreases, but the amplitude increases drastically, leading to a devastatingly powerful wave impact against the shoreline and coastal civilization. Any analysis of these phenomena can be useful in the effort to minimize the potentially catastrophic effects of future tsunamis. 
An excellent way to aid this effort is to understand and predict mathematically how a tsunami will behave during its run-up and draw-down on the shoreline. Exact analytic solutions have been previously found for nonlinear non-breaking shallow water waves on a constant-sloped beach [4]] [10]. These findings, while accurate, are difficult to represent in a concise, concrete fashion and consequently are extremely challenging to use in computational applications. In our investigation we attempt to accurately predict the same behavior using a simpler model. A key feature of our solution, which makes it especially relevant in real-world application, is that it can easily be adapted to any piecewise-linear configuration of the beach floor. While we primarily discuss our results for a consant-sloped configuration in order to allow easy comparison with the majority of previous literature, the ability to model the wave behavior for differing seafloor patterns allows engineers to test possible barriers and perhaps determine methods to minimize the effects of tsunamis on the coast.

To find our solutions, we first non-dimensionalize the nonlinear shallow-water wave equations and make a substitution to simplify our initial problem. Applying the method of perturbations by using the small parameter $\varepsilon$ and dropping terms with high powers of this parameter allows us to obtain the desired simplification. The resulting system can be solved using an integral transformation; this yields our approximate analytic solution, the accuracy of which is tested successfully against a numerical approximation. The increased simplicity of our solution will help make moving boundary problems more accessible and might help in the effort to protect coastal regions from tsunami destruction.

\subsection{Problem Statement}

The following diagram illustrates the set-up of our problem and the most essential variables.

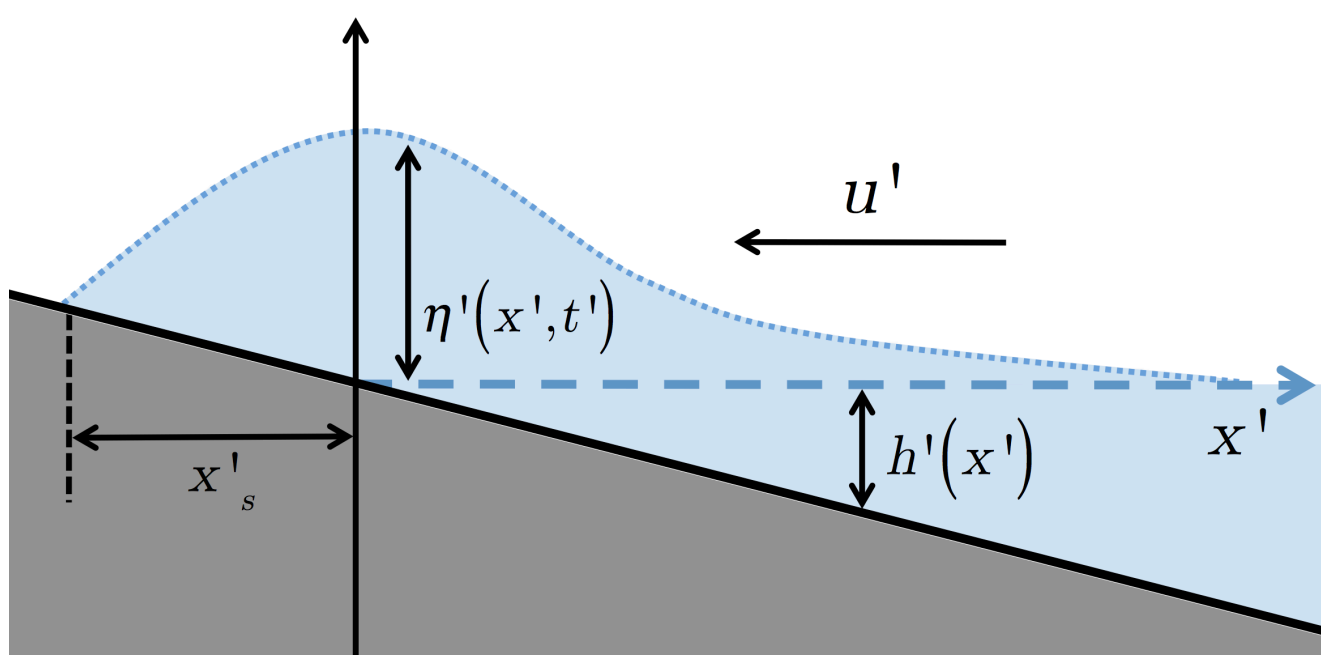

Figure 1: Schematic of a wave approaching a constant-sloped beach.

The dotted line represents the moving water surface and the solid diagonal 
line marks the ocean floor. Its important to note that the superscript ' denotes a dimensional variable (i.e. a variable with units of measurement) rather than a derivative. As the wave moves up the beach, its amplitude, $\eta^{\prime}$, increases while the fluid velocity, $u^{\prime}$ decreases. The beach is assumed to have constant slope $-\alpha$ (the choice of $-\alpha$ rather than $\alpha$ is simply for ease of calculation later). At the point $x^{\prime}=0$, the height of the beach is equal to that of the initial sea level (marked by the dashed line). The relevant nomenclature for our problem is summarized in Table 1 (with primes excluded).

Table 1: Description of Relevant Variables

\begin{tabular}{|c|l|}
\hline Variables & \multicolumn{1}{|c|}{ Descriptions } \\
\hline$x$ & Horizontal position coordinate, positive to the right of $x=0$ \\
\hline$t$ & Time \\
\hline$\eta(x, t)$ & Amplitude of wave above neutral ocean height \\
\hline$u(x, t)$ & Horizontal fluid velocity \\
\hline$\varphi(x)=h(x)$ & Vertical distance from ocean surface to ocean floor at position $x$ \\
\hline$\lambda$ & Characteristic wavelength \\
\hline$x_{s}(t)$ & Horizontal position coordinate of the moving shoreline, positive to the left of $x=0$ \\
\hline$f(x)$ & Equation modeling initial wave form \\
\hline$-\alpha$ & The constant slope of the shoreline \\
\hline$\varepsilon=\frac{\eta_{0}}{h_{0}}$ & The ratio of characteristic wave height to still-water depth \\
\hline$g$ & Gravitational constant \\
\hline
\end{tabular}

We recall that for a tsunami, the wavelength is significantly greater than the ocean depth, whereas the characteristic amplitude is extremely small. This allows us (in keeping with previous literature) to use the shallow-water wave equations, a system of partial differential equations given by (1) and (2)[[9][11] These well-documented equations are derived from the more general NavierStokes equations by scale analysis, eliminating some terms such as bottom friction whose effects are minimized in the shallow-water scenario. Due to the nature of tsunamis they can be modelled using the shallow-water equations even in the middle of the ocean (and certainly close to shore as in the present case). We begin with the more general nonlinear form of these equations rather than the simplified linear version used in some literature.

$$
\begin{aligned}
\frac{\partial u^{\prime}}{\partial t^{\prime}}+u^{\prime} \frac{\partial u^{\prime}}{\partial x^{\prime}}+g \frac{\partial \eta^{\prime}}{\partial x^{\prime}} & =0 \\
\frac{\partial \eta^{\prime}}{\partial t^{\prime}}+\frac{\partial}{\partial x}\left[\left(\eta^{\prime}+\varphi^{\prime}\right) u^{\prime}\right] & =0
\end{aligned}
$$

To complete our problem, we add initial and boundary conditions. We assume the wave begins with a particular shape given by $f(x)$ while fluid is 
initially stationary, as shown in equations (3) and (4). Since the tsunami is a local phenomenon, the amplitude and velocity of the wave should limit to 0 as $x$ approaches infinity, as shown in equations (5) and (6).

$$
\begin{gathered}
t^{\prime}=0, \quad \eta^{\prime}\left(x^{\prime}, 0\right)=f^{\prime}\left(x^{\prime}\right) \\
t^{\prime}=0, \quad u^{\prime}\left(x^{\prime}, 0\right)=0 \\
\lim _{x^{\prime} \rightarrow \infty} \eta^{\prime}\left(x^{\prime}, t^{\prime}\right)=0 \\
\lim _{x^{\prime} \rightarrow \infty} u^{\prime}\left(x^{\prime}, t^{\prime}\right)=0
\end{gathered}
$$

Finally, we can find the moving shoreline $x_{s}$ implicitly by finding the point at which $\eta=\varphi$ (since $\varphi$ represents the beach for all $x$ ). Note that $x_{s}$ is thus dependent on $t$ and represents the moving boundary of our model.

$$
x^{\prime}=-x_{s}^{\prime}\left(t^{\prime}\right), \quad \eta^{\prime}\left(-x_{s}^{\prime}\left(t^{\prime}\right), t^{\prime}\right)=-\varphi^{\prime}\left(-x_{s}^{\prime}\left(t^{\prime}\right)\right)
$$

\section{Solution}

\subsection{Non-Dimensional Model}

We begin by nondimensionalizing the variables of our problem, thereby eliminating the need to note the units of an equation. To do so, we divide each dimensional variable by its corresponding characteristic value (denoted by the subscript ${ }_{0}$ ), as shown in the following substitutions. The magnitudes of $\eta_{0}$ and $h_{0}$ are known from the initial assumptions of the problem.

$$
t=\frac{t^{\prime}}{t_{0}}, \quad u=\frac{u^{\prime}}{u_{0}}, \quad x=\frac{x^{\prime}}{x_{0}}, \quad \eta=\frac{\eta^{\prime}}{\eta_{0}}, \quad \varphi=\frac{\varphi^{\prime}}{h_{0}} .
$$

We further simplify our non-dimensional model by using scale analysis on equations (1) and (2) [[5], [[8].

$$
t_{0}=\frac{\ell_{0}}{c_{0}}, \quad c_{0}=\sqrt{g h_{0}}, \quad u_{0}=\eta_{0} \sqrt{\frac{g}{h_{0}}} .
$$

Applying the above substitutions to equations (1) and (2) yields the following non-dimensional equations, where $\varepsilon=\eta_{0} / h_{0}$ :

$$
\begin{gathered}
\frac{\partial u}{\partial t}+\varepsilon u \frac{\partial u}{\partial x}+\frac{\partial \eta}{\partial x}=0, \\
\frac{\partial \eta}{\partial t}+\frac{\partial}{\partial x}[u(\varphi(x)+\varepsilon \eta)]=0 .
\end{gathered}
$$

Finally, applying the same substitutions to our initial and boundary conditions yields these corresponding non-dimensional initial and boundary conditions: 


$$
\begin{gathered}
t=0, \quad \eta(x, 0)=f(x) ; \\
t=0, \quad u(x, 0)=0 ; \\
\lim _{x \rightarrow \infty} \eta(x, t)=0 ; \\
\lim _{x \rightarrow \infty} u(x, t)=0 ; \\
x=-x_{s}(t), \quad \varepsilon \eta\left(-x_{s}, t\right)=-\varphi\left(-x_{s}\right) .
\end{gathered}
$$

\subsection{Eliminating the Moving Boundary}

Now that we have non-dimensionalized our model, we look to eliminate the primary difficulty of our system of equations: the moving boundary $x_{s}$. As the wave approaches and recedes from the shore, $x_{s}$ is continuously changing, which complicates the solution to our system since the boundary for the independent variable $x$ is given by $x \geq x_{s}$. Instead of attempting to solve our problem in terms of that moving boundary, we make the following substitutions:

$$
x_{m}=x_{s} / \varepsilon, \quad x=y-\varepsilon x_{m} .
$$

The new variable $x_{m}$ is simply a rescaling of the variable $x_{s}$ (necessary to give the unknown variables the same order with respect to $\varepsilon$ ), and replacing the spatial variable $x$ with the new variable $y$ changes the computational domain from the moving boundary $x \geq x_{s}$ to the fixed $y \geq 0$. Substituting these into equations (8)-(14) yields the following modified system of differential equations:

$$
\begin{gathered}
\frac{\partial u}{\partial t}+\frac{\partial \eta}{\partial y}+\varepsilon \frac{\partial u}{\partial y}\left(u-\dot{x}_{m}\right)=0 \\
\frac{\partial \eta}{\partial t}+\frac{\partial}{\partial y}\left(u \varphi\left(y-\varepsilon x_{m}\right)\right)+\varepsilon \frac{\partial}{\partial y}\left[\eta\left(u-\dot{x}_{m}\right)\right]=0 \\
t=0, \quad \eta(y, 0)=f(y) \\
t=0, \quad u(y, 0)=0 \\
\lim _{y \rightarrow \infty} \eta(y, t)=0 \\
\lim _{y \rightarrow \infty} u(y, t)=0 \\
y=0, \quad \varepsilon \eta\left(-\varepsilon x_{m}, t\right)=-\varphi\left(-\varepsilon x_{m}\right)
\end{gathered}
$$

Note that a dot over a variable, such as $\dot{\varphi}$, denotes the first derivative of that variable, and multiple dots denote the corresponding-order derivative. This notation is used only for functions of a single independent variable.

We can simplify the term $\varphi\left(y-\varepsilon x_{m}\right)$ by applying the Taylor series approximation about $y$. The general approximation for some arbitrary variable $z$ about the point $y$ is 


$$
\varphi(z)=\varphi(y)+\dot{\varphi}(y)(z-y)+\dot{\varphi}(y)(z-y)^{2} / 2+\ldots
$$

Translating this into an approximation for $y-\varepsilon x_{m}$ about $y$ gives

$$
\varphi\left(y-\varepsilon x_{m}\right)=\varphi(y)+\dot{\varphi}(y)\left(-\varepsilon x_{m}\right)+O\left(\varepsilon^{2}\right) .
$$

Recall that the parameter $\varepsilon$ represents the ratio of characteristic wave height to characteristic ocean depth, and it is thus extremely small. In fact, a real-world tsunami typically has an amplitude around just $30 \mathrm{~cm}$, whereas the characteristic depth of the ocean is approximately $3.7 \mathrm{~km}$ (and hence $\varepsilon \approx 0.00000008$ ). Given this extremely small scale, we simplify the system by dropping any terms with order of $\varepsilon^{2}$ or higher [9] [11]

Substituting the Taylor series approximation into (16) gives us

$$
\frac{\partial \eta}{\partial t}+\frac{\partial}{\partial y}\left[u\left(\varphi(y)-\varepsilon \dot{\varphi}(y) x_{m}\right)\right]+\varepsilon \frac{\partial}{\partial y}\left[\eta\left(u-\dot{x}_{m}\right)\right]=0 .
$$

We regroup this equation so that the $\varepsilon$ terms are shown together:

$$
\frac{\partial \eta}{\partial t}+\frac{\partial}{\partial y}[u \varphi(y)]+\varepsilon \frac{\partial}{\partial y}\left[\eta\left(u-\dot{x}_{m}\right)-u \dot{\varphi}(y) x_{m}\right]=0 .
$$

In a similar manner, we can rewrite our final boundary condition (21) using Maclaurin expansions for $\eta$ and $\varphi$ about $y=0$.

$$
\begin{gathered}
\varepsilon\left(\eta(0, t)-\left.\varepsilon \frac{\partial \eta}{\partial y}\right|_{y=0} x_{m}+O\left(\varepsilon^{2}\right)\right)=-\left(\varphi(0)-\varepsilon \dot{\varphi}(0) x_{m}+\frac{\dot{\varphi}(0) \varepsilon^{2} x_{m}^{2}}{2}+O\left(\varepsilon^{3}\right)\right) \\
\eta(0, t)-\left.\varepsilon \frac{\partial \eta}{\partial y}\right|_{y=0} x_{m}=\dot{\varphi}(0) x_{m}-\varepsilon \frac{\dot{\varphi}(0) x_{m}^{2}}{2}
\end{gathered}
$$

Since we know that

$$
\dot{\varphi}(0)=-\alpha, \quad: \varphi(0)=0
$$

this simplifies to

$$
\eta(0, t)-\left.\varepsilon \frac{\partial \eta}{\partial y}\right|_{y=0} x_{m}=-\alpha x_{m}
$$




\subsection{Method of Perturbations}

While we have simplified our problem somewhat by non-dimensionalizing and eliminating the moving boundary, we here perform our most significant simplification by applying the method of perturbations to the unknown functions $\eta, u$, and $x_{m}$, utilizing the small parameter $\varepsilon(\approx 0.00000008)$. This allows us to diverge from previous findings by seeking an approximate analytic solution rather than an exact solution. This approximate solution will still be obtained in such a way as to remain effectively accurate, but as we shall see the results are significantly simpler computationally. Note that we are now using the subscript 0 to denote the first term in our approximations rather than a characteristic value. We expand our variables as

$$
\eta=\eta_{0}+\varepsilon \eta_{1}+O\left(\varepsilon^{2}\right), \quad u=u_{0}+\varepsilon u_{1}+O\left(\varepsilon^{2}\right), \quad x_{m}=x_{0}+\varepsilon x_{1}+O\left(\varepsilon^{2}\right) .
$$

Substituting into equations (15)-(20),(27) yields:

$$
\begin{gathered}
\frac{\partial u_{0}}{\partial t}+\varepsilon \frac{\partial u_{1}}{\partial t}+\frac{\partial \eta_{0}}{\partial y}+\varepsilon \frac{\partial \eta_{1}}{\partial y}-\varepsilon \frac{\partial u_{0}}{\partial y} \dot{x}_{0}+\varepsilon u_{0} \frac{\partial u_{0}}{\partial y}=0 \\
\frac{\partial \eta_{0}}{\partial t}+\varepsilon \frac{\partial \eta_{1}}{\partial t}+\frac{\partial\left(u_{0} \varphi(y)\right)}{\partial y}+\varepsilon \frac{\partial}{\partial y}\left[u_{1} \varphi(y)-u_{0} \dot{\varphi}(y) x_{0}+\eta_{0}\left(u_{0}-\dot{x_{0}}\right)\right]=0 \\
t=0, \quad \eta_{0}(y, 0)+\varepsilon \eta_{1}(y, 0)=f(y) ; \\
t=0, u_{0}(y, 0)+\varepsilon u_{1}(y, 0)=0 ; \\
\lim _{y \rightarrow \infty} \eta_{0}(y, t)+\varepsilon \eta_{1}(y, t)=0 ; \\
\lim _{y \rightarrow \infty} \lim _{0} u_{0}(y, t)+\varepsilon u_{1}(y, t)=0 ; \\
\eta_{0}(0, t)+\varepsilon \eta_{1}(0, t)-\left.\varepsilon \frac{\partial \eta_{0}}{\partial y}\right|_{y=0} x_{0}=-\alpha x_{0}-\varepsilon \alpha x_{1} .
\end{gathered}
$$

Since $\varepsilon$ is arbitrary, we can separate equations (28), (29), and (34) by the terms with or without $\varepsilon$.

$$
\begin{gathered}
\frac{\partial u_{0}}{\partial t}+\frac{\partial \eta_{0}}{\partial y}=0 \\
\frac{\partial \eta_{0}}{\partial t}+\frac{\partial}{\partial y}\left(u_{0} \varphi(y)\right)=0 \\
\eta_{0}(0, t)+\alpha x_{0}=0 \\
\frac{\partial \eta_{1}}{\partial t}+\frac{\partial \eta_{1}}{\partial y}+\left(u_{0}-\dot{x}_{0}\right) \frac{\partial u_{0}}{\partial y}=0, \\
\frac{\partial y}{\partial y}\left[u_{1} \varphi(y)-u_{0} \dot{\varphi}(y) x_{0}+\eta_{0}\left(u_{0}-\dot{x}_{0}\right)\right]=0 \\
\eta_{1}(0, t)-\left.\frac{\partial \eta_{0}}{\partial y}\right|_{y=0} x_{0}+\alpha x_{1}=0 .
\end{gathered}
$$


Equations (35)-(37) are drawn from terms with no $\varepsilon$, and contain the functions $\eta_{0}, u_{0}$, and $x_{0}$, whereas (38)-(40) are drawn from terms multiplied by an $\varepsilon$ term, and contain the functions $\eta_{1}, u_{1}$, and $x_{1}$. Our focus in this paper is on solving for the former three functions. We start by similarly separating the initial and boundary conditions by $\varepsilon$.

$$
\begin{gathered}
t=0, \quad \eta_{0}(y, 0)=f(y) ; \\
\lim _{y \rightarrow \infty} \eta_{0}(y, t)=0 ; \\
t=0, \quad u_{0}(y, 0)=0 ; \\
\lim _{y \rightarrow \infty} u_{0}(y, t)=0 ; \\
t=0, \quad \eta_{1}(y, 0)=f(y) ; \\
\lim _{y \rightarrow \infty} \eta_{1}(y, t)=0 ; \\
t=0, \quad u_{1}(y, 0)=0 ; \\
\lim _{y \rightarrow \infty} u_{1}(y, t)=0 .
\end{gathered}
$$

We use an algebraic elimination to find a differential equation for $\eta_{0}$. From (35), we know that:

$$
\frac{\partial u_{0}}{\partial t}=-\frac{\partial \eta_{0}}{\partial y} .
$$

Taking the derivative of (36) with respect to $t$ and substituting yields:

$$
\begin{aligned}
\frac{\partial^{2} \eta_{0}}{\partial t^{2}}+\frac{\partial}{\partial t} \frac{\partial}{\partial y}\left(u_{0} \varphi(y)\right) & =0 \\
\frac{\partial^{2} \eta_{0}}{\partial t^{2}}+\frac{\partial}{\partial y}\left(\frac{\partial u_{0}}{\partial t} \varphi(y)\right) & =0 \\
\frac{\partial^{2} \eta_{0}}{\partial t^{2}}-\frac{\partial}{\partial y}\left(\frac{\partial \eta_{0}}{\partial y} \varphi(y)\right) & =0 .
\end{aligned}
$$

We now have found a second-order differential equation for $\eta_{0}$. Using (50) allows us to derive an additional initial condition. Since

$$
\begin{gathered}
\frac{\partial \eta_{0}}{\partial t}=-\frac{\partial}{\partial y}\left(u_{0} \varphi(y)\right), \\
\frac{\partial \eta_{0}}{\partial t}=-\frac{\partial u_{0}}{\partial y} \varphi(y)-u_{0} \dot{\varphi}(y),
\end{gathered}
$$

using initial condition (43) on $u_{0}$ and (49), we can conclude that

$$
\left.\frac{\partial \eta_{0}}{\partial t}\right|_{t=0}=0 .
$$




\subsection{Integral Transform}

We will now solve equations (41),(42),(51),(52) for $\eta_{0}$ using an integral transformation. For our chosen transformation we approximate the infinite boundary with the constant $L$. We define $\hat{\eta}_{0}$ as follows, where the kernel $K(y, \lambda)$ is an unspecified function:

$$
\hat{\eta}_{0}(\lambda, t)=\int_{0}^{L} \eta_{0}(\lambda, t) K(y, \lambda) d y .
$$

We impose the following restrictions on $K$ :

$$
\begin{gathered}
K(L)=0 \\
\frac{d}{d y}\left(\varphi(y) \frac{d K}{d y}\right)=-\lambda^{2} K .
\end{gathered}
$$

Multiplying the equation for $n_{0},(51)$, by $K$ and integrating with respect to $y$ yields that:

$$
\begin{gathered}
\int_{0}^{L} \frac{\partial^{2} \eta_{0}}{\partial t^{2}} K(y, \lambda) d y=\int_{0}^{L} \frac{\partial}{\partial y}\left(\frac{\partial \eta_{0}}{\partial y} \varphi(y)\right) K(y, \lambda) d y \\
\frac{\partial^{2} \hat{\eta}_{0}}{\partial t^{2}}=\int_{0}^{L} \frac{\partial}{\partial y}\left(\frac{\partial \eta_{0}}{\partial y} \varphi(y)\right) K(y, \lambda) d y .
\end{gathered}
$$

From here, we may use integration by parts to see that

$$
\begin{gathered}
\frac{\partial^{2} \hat{\eta}_{0}}{\partial t^{2}}=\left.\left(\frac{\partial \eta_{0}}{\partial y} \varphi(y) K(y, \lambda)\right)\right|_{0} ^{L}-\int_{0}^{L} \frac{\partial \eta_{0}}{\partial y} \varphi(y) \frac{d K}{d y} d y \\
\frac{\partial^{2} \hat{\eta}_{0}}{\partial t^{2}}=\int_{0}^{L} \eta_{0} \frac{d}{d y}\left(\varphi(y) \frac{d K}{d y}\right) d y
\end{gathered}
$$

and making some final substituions using (54) and (53) yields

$$
\frac{\partial^{2} \hat{\eta}_{0}}{\partial t^{2}}=-\lambda^{2} \hat{\eta}_{0} .
$$

We now have a second-order differential equation for $\hat{\eta}_{0}$. Similarly transforming our initial conditions (41) and (52) on $\eta_{0}$ yields

$$
\begin{gathered}
\hat{\eta}_{0}(\lambda, 0)=\hat{f}(\lambda):=\int_{0}^{L} f(y) K(y, \lambda) d y, \\
\left.\frac{\partial \hat{\eta}_{0}}{\partial t}\right|_{t=0}=0 .
\end{gathered}
$$

Equation (55) is of course well-known to even elementary students of differential equations, with the resulting general solution for $\hat{\eta}$ being 


$$
\hat{\eta}_{0}(\lambda, t)=A \cos (\lambda t)+B \sin (\lambda t) .
$$

Using our initial conditions for $\hat{\eta}_{0}$, we must have that

$$
\hat{\eta}_{0}(\lambda, t)=\hat{f}(\lambda) \cos (\lambda t) .
$$

Now that we have found $\hat{\eta}_{0}$, we turn our attention to finding $K$. We know that $\varphi(y)=-\alpha y$, so we can substitute into (54):

$$
\frac{d}{d y}\left(-\alpha y \frac{d K}{d y}\right)=-\lambda^{2} K .
$$

We now let $z=\sqrt{y}$, and substitute into (59):

$$
\begin{gathered}
-\alpha \frac{1}{2 z} \frac{d}{d z}\left(z^{2} \frac{1}{2 z} \frac{d K}{d z}\right)=-\lambda^{2} K \\
\frac{d}{d z}\left(z \frac{d K}{d z}\right)=\frac{4 \lambda^{2}}{\alpha} z K .
\end{gathered}
$$

This equation has a known solution (see [12]]. If $J_{i}$ denotes the Bessel Function or order $i$, our solution is

$$
K\left(y, \lambda_{k}\right)=\frac{\sqrt{2}}{\sqrt{L}} \frac{J_{0}\left(\sqrt{\frac{4 \lambda_{k}^{2}}{\alpha} z}\right)}{\left|J_{1}\left(\sqrt{\frac{4 \lambda_{k}^{2} L}{\alpha}}\right)\right|} .
$$

This solution is valid only for a countably infinite set of values of $\lambda$, indexed by $\lambda_{k}$. These are defined by [2]:

$$
J_{0}\left(\sqrt{\frac{4 \lambda_{k}^{2} L}{\alpha}}\right)=0 .
$$

Having obtained solutions for $\hat{\eta}_{0}(58)$ and $K(61)$, Sturm-Liouville theory uses the orthonormality and the countable cardinality of our eigenfunctions $K\left(y, \lambda_{k}\right)$ to obtain the inversion formula [12];

$$
\begin{gathered}
\eta_{0}(y, t)=\sum_{k=1}^{\infty} \hat{\eta}_{0}\left(\lambda_{k}, t\right) K\left(y, \lambda_{k}\right) \\
\eta_{0}(y, t)=\sum_{k=1}^{\infty}\left[\hat{f}\left(\lambda_{k}\right) \cos \left(\lambda_{k} t\right) \frac{\sqrt{2}}{\sqrt{L}} \frac{J_{0}\left(\sqrt{\frac{4 \lambda_{k}^{2} y}{\alpha}}\right)}{\left|J_{1}\left(\sqrt{\frac{4 \lambda_{k}^{2} L}{\alpha}}\right)\right|}\right] .
\end{gathered}
$$


This equation presents $\eta_{0}$ as function of $x$ and $t$ in closed and relatively simple form, as we had hoped. This is the function we use to model the behavior of a tsunami wave approaching an infinitely long constant-sloped beach. Using this solution with (35) gives an equation for $u_{0}$ :

$$
\begin{gathered}
\frac{\partial u_{0}}{\partial t}=-\sum_{k=1}^{\infty}\left[\hat{f}\left(\lambda_{k}\right) \cos \left(\lambda_{k} t\right) \sqrt{\frac{2 \lambda_{k}^{2}}{\alpha L y}} \frac{-J_{1}\left(\sqrt{\frac{4 \lambda_{k}^{2} y}{\alpha}}\right)}{\left|J_{1}\left(\sqrt{\frac{4 \lambda_{k}^{2} L}{\alpha}}\right)\right|}\right] \\
u_{0}(y, t)=\sum_{k=1}^{\infty}\left[\hat{f}\left(\lambda_{k}\right) \sin \left(\lambda_{k} t\right) \sqrt{\frac{2}{\alpha L y}} \frac{J_{1}\left(\sqrt{\frac{4 \lambda_{k}^{2} y}{\alpha}}\right)}{\left|J_{1}\left(\sqrt{\frac{4 \lambda_{k}^{2} L}{\alpha}}\right)\right|}\right]+\psi(y)
\end{gathered}
$$

where $\psi(y)$ is some arbitrary function. However, we recall our initial condition (43), that $u_{0}(y, 0)=0$. Therefore $\psi(y)=0$, and we can simplify (64):

$$
u_{0}(y, t)=\sum_{k=1}^{\infty}\left[\hat{f}\left(\lambda_{k}\right) \sin \left(\lambda_{k} t\right) \sqrt{\frac{2}{\alpha L y}} \frac{J_{1}\left(\sqrt{\frac{4 \lambda_{k}^{2} y}{\alpha}}\right)}{\left|J_{1}\left(\sqrt{\frac{4 \lambda_{k}^{2} L}{\alpha}}\right)\right|}\right]
$$

We have now found equations for both $\eta_{0}$ and $u_{0}$, and using (37) (which states that $\left.\eta_{0}(0, t)+\alpha x_{0}=0\right)$ and the fact that $J_{0}(0)=1[[2]$, we can solve for $x_{0}$ :

$$
x_{0}(t)=\sum_{k=1}^{\infty}\left[\frac{\hat{f}\left(\lambda_{k}\right) \sqrt{2} \cos \left(\lambda_{k} t\right)}{-\alpha \sqrt{L}\left|J_{1}\left(\sqrt{\frac{4 \lambda_{k}^{2} L}{\alpha}}\right)\right|}\right]
$$

We have now obtained final solutions for $\eta_{0}, x_{0}$, and $u_{0}$ as we desired.

\section{Analysis}

\subsection{Results}

Now that we have solved our system to obtain explicit functions for $\eta_{0}, u_{0}$, and $x_{0}$, we begin verifying the accuracy of our function by plotting it (thereby ensuring that it passes the 'eye test'). To plot our function $\eta_{0}$ we used Wolfram Mathematica, a well-known mathematical and computational program. Note that, since our solution is in the form of an infinite series, we obtained graphs by truncating the series. The graphs obtained by truncating the series at anywhere from 100 to 5000 are visually identical, differing by approximately 0.001 , which suggests that the series converges extremely quickly and that our truncated plots reflect almost exactly the behavior of the full solution.

Firstly, in figure 2 we plot our solution for the wave amplitude $\eta_{0}$ at several different points in time. 


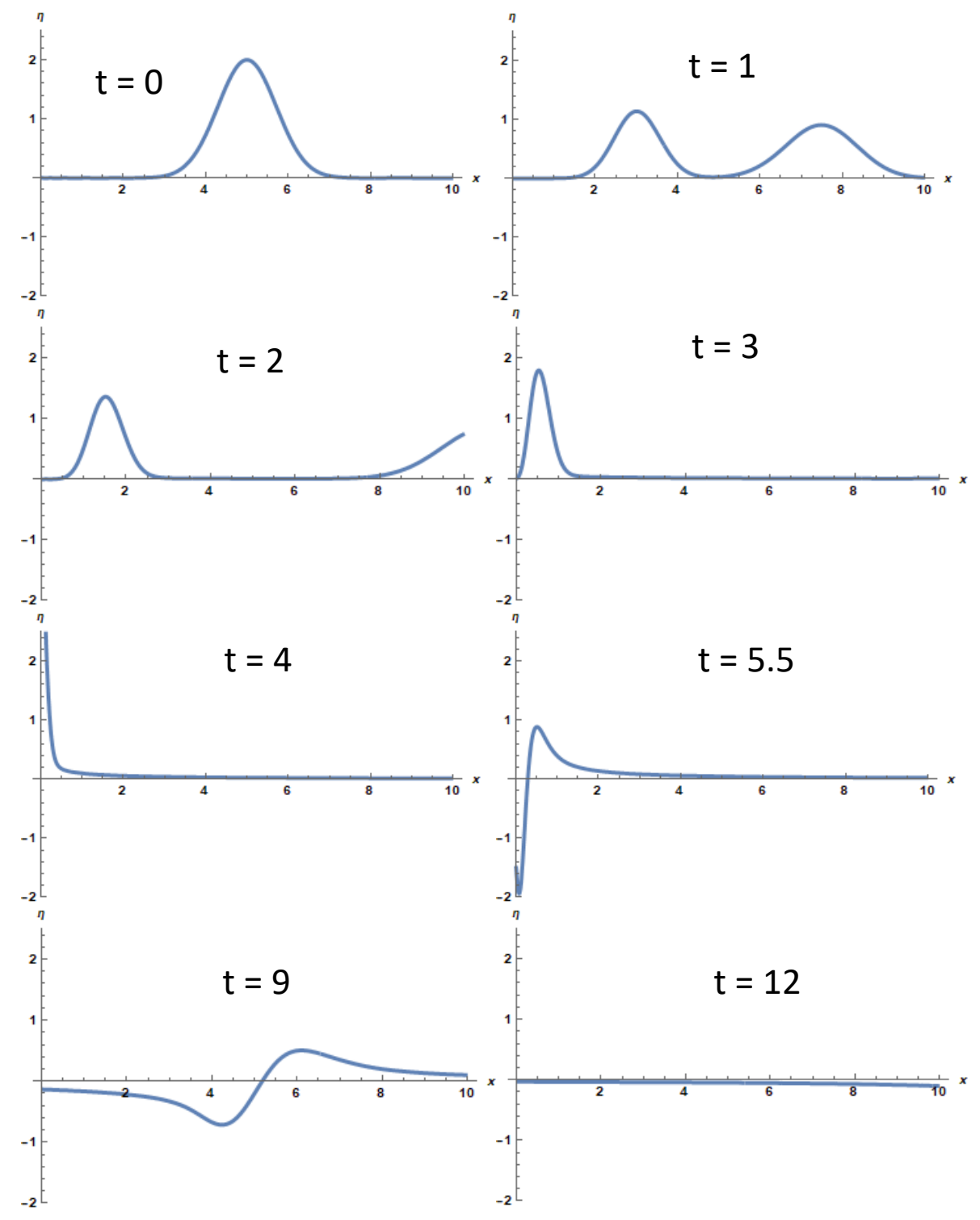

Figure 2: The wave-form (our solution) at different points in time.

To continue testing the validity of our analytical solution for $\eta_{0}$, we compare our plot to a numerical approximation obtained through Mathematica. We use our second-order differential equation for $\eta_{0}$ (51) and the relevant initial conditions (41),(52) as our Mathematica input to be solved for and plotted. Although the numerical approximation is not an exact solution, it has a high degree of accuracy and thus acts as a useful comparison. While it would perhaps be even more informative to compare our results to a numerical solution to the original equations (1) and (2), obtaining numerical solutions to these equations 
is a more challenging problem which proved beyond the scope of our research. In figure 3, we plot the difference between our solution and the numeric solution at various points in time.

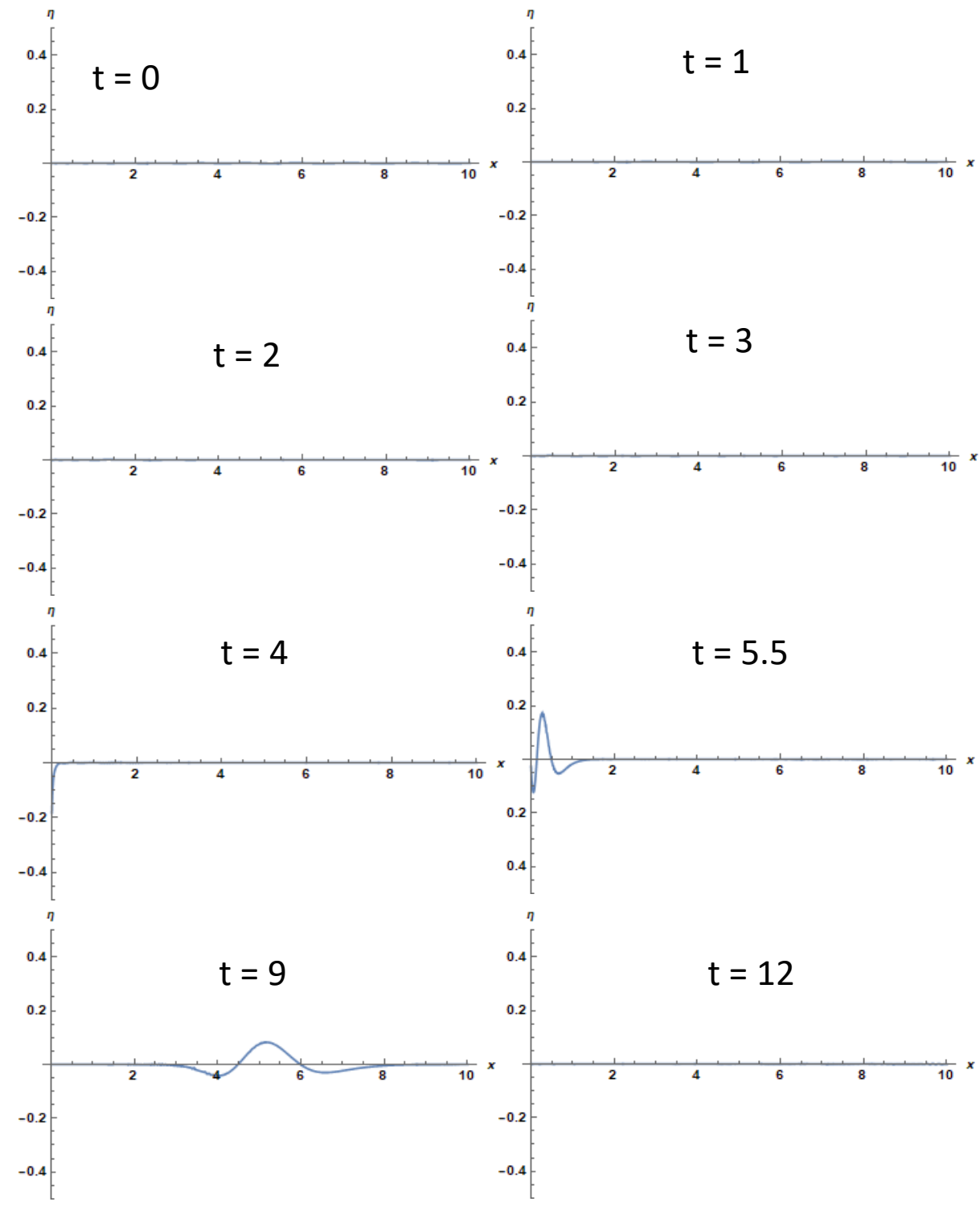

Figure 3: The difference between the our solution and the numeric solution at various points in time (note the change in scale from the previous figure). 


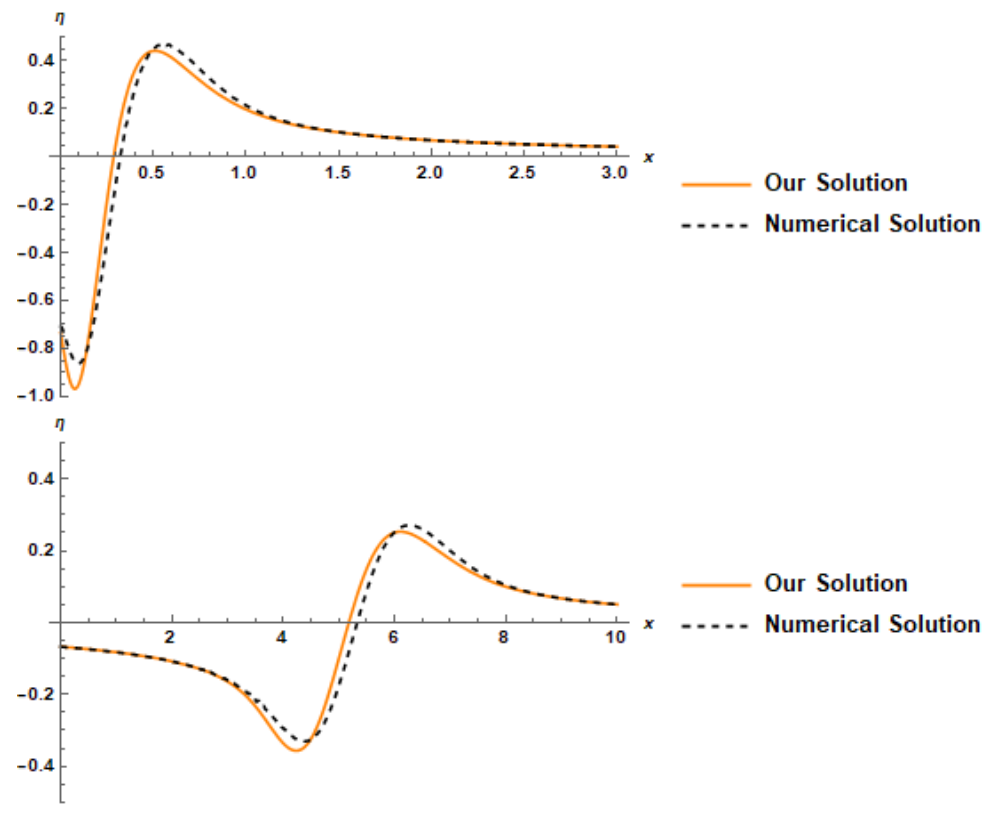

Figure 4: Comparison of the two solutions at $t=5.5$ and $t=9$.

As figure 3 suggests, the two solutions agree very closely at most points in time, but some disparity occurs during wave draw-down, as seen for $t=5.5$ and $t=9$. Figure 4 shows the wave-form according to both solutions at those points in time. Comparing these plots, we can see that the disparity which occurs is a result of the numerical wave drawing down from the beach slightly ahead of our wave.

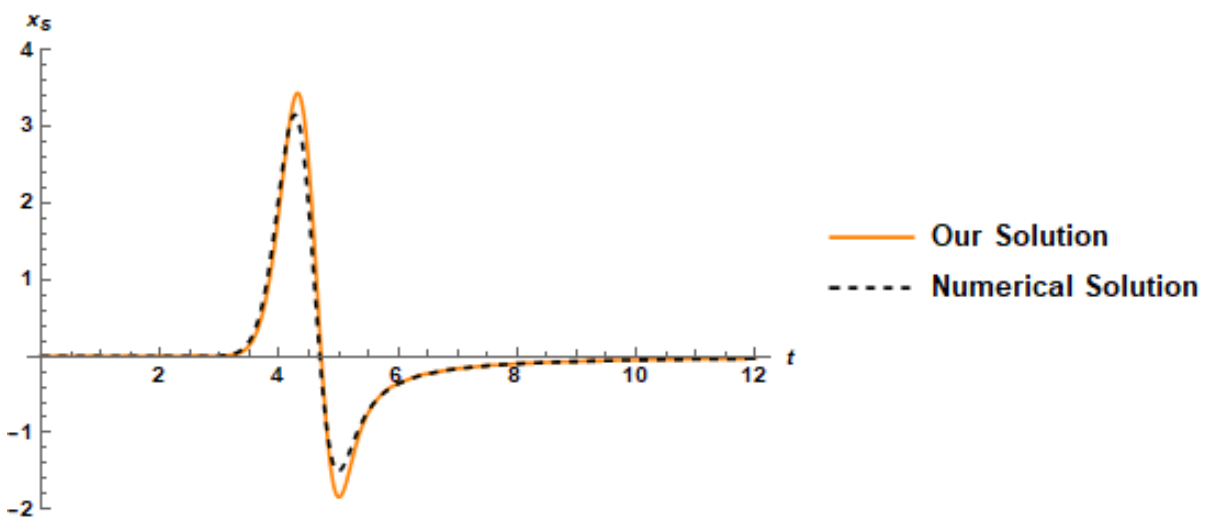

Figure 5: The movement of the shoreline point according to the two solutions.

To help understand this disparity, we plot in figure 5 the movement of the shoreline $\left(x_{s}\right)$ over time according to the two solutions. We can see from this 
that the numerical wave drawing-down slightly earlier is a consequence of the numerical solution estimating slightly less movement of the shoreline, effectively predicting the wave to peak slightly earlier. It's likely that this variation between solutions can be attributed to the well-known tendency of numerical approximations such as that used by Mathematica to smoothen the most extreme behaviors of the solutions. As we can see from figure 3, the slight lag of the draw-down wave is the only perceptible disparity between the solutions (for example, the average error at $t=3$ is 0.000117 ). The fact that these solutions are so similar is a good indicator that our explicit analytical solution for $\eta_{0}$ is accurate.

In addition to two-dimensional plots, we can also visualize our solution in three dimensions. Using Mathematica, we graph our explicit analytical solution for the tsunami wave in three-dimensions (Figure 6), representing time as a second variable axis.
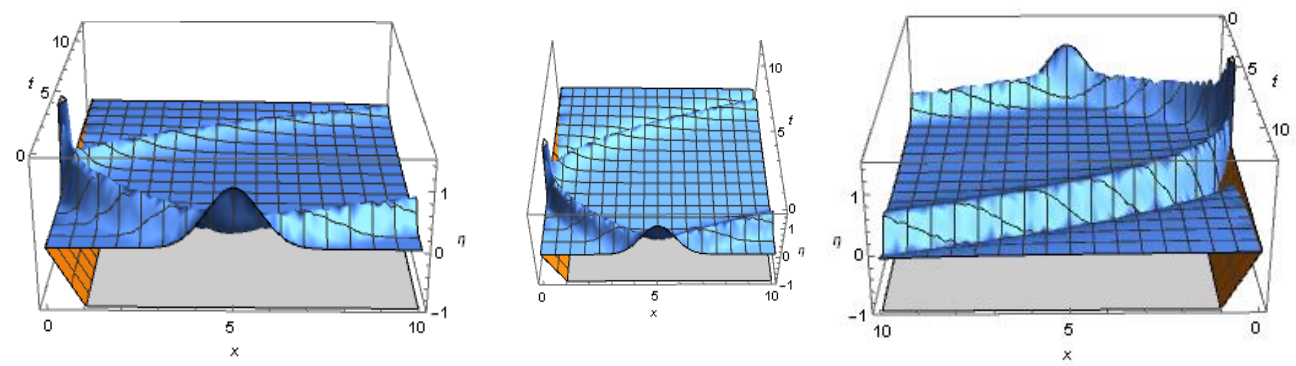

Figure 6: Three-dimensional graph of our solution.

The three-dimensional graphs shows how the wave behaves for $0 \leq t \leq 10$ simultaneously. The vertical axis measures the wave amplitude, with the horizontal axis being the spatial variable and the depth axis time. At $t=0$ we can see the initial wave profile (most clearly visible in the leftmost graph), which splits as always into a seaward wave and a shorebound wave. From the threedimensional graph, we clearly see that as the wave approaches the shore, the rise in amplitude is accompanied by a decrease in velocity, as evidenced by the curved shape of the waveform visible in the second graph. After the breaking point occurs, we can see the draw-down wave undergo the reverse phenomenon, as a gradual decline in amplitude is accompanied by acceleration as the wave moves back out to sea. The rightmost graph more clearly depicts the decreasing amplitude of the draw-down wave as well as allowing a look into the wave trough.

To further test the validity of our analytical solution, in Figure 7 we compare our three-dimensional graph to the three-dimensional graph of the same numerical approximation used earlier. From this graph we can see as in Figure 3 that the two solutions effectively coincide, with the exception of the numerical wave drawing-down slightly ahead of our wave. Once again, we see that a comparison with the numerical solution suggests our explicit solution is indeed accurate. 

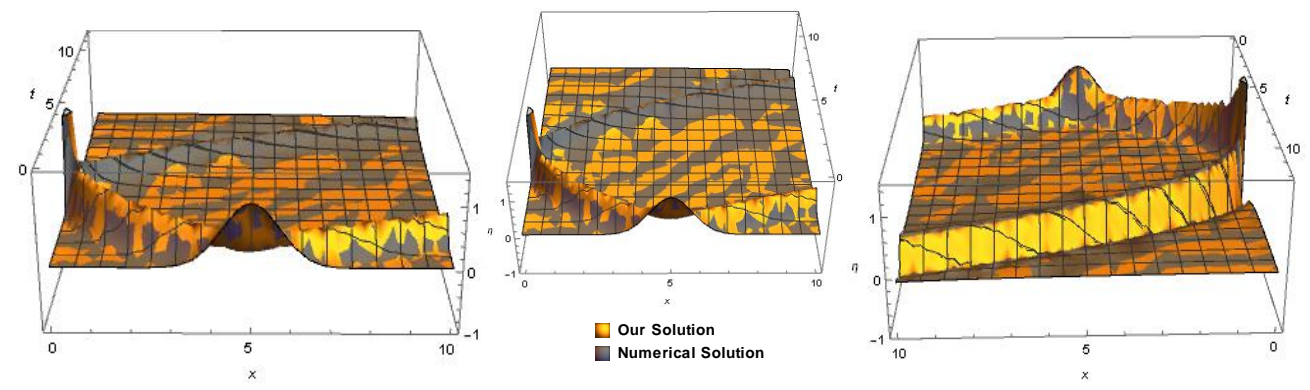

Figure 7: Three-dimensional comparison of the two solutions.

\subsection{Conclusions}

We set out to find a model for wave run-up and draw-down that was simpler than those of previous research. After the first steps of non-dimensionalization and eliminating the moving boundary, our usage of the method of perturbations allowed us to do so, yielding a relatively simple system which proved solvable using a single integral transformation.

Mathematica verified that our solutions were computationally simple; we were able to plot them within seconds using only standard computational packages. The program also enabled us to verify the accuracy of our solution, comparing the resulting plots to a numerical solution. Since the only (minor) variation appears to be a natural result of the smoothening tendencies of the numeric approximation, we conclude that our results are also effectively accurate.

Our ability to express tsunami wave behavior in a computationally accessible form could aid future civil engineering projects in protecting coastline regions from the destruction of tsunamis. Further aiding future projects is the potential to adapt our solution to different seafloor configurations. In addition, our efficient process and explicit expression of the solution will help make moving boundary problems more accessible for civil projects and further research.

From a mathematical perspective, there are many opportunities for future researchers to build upon this work. Two such opportunities appear particularly promising. First, when applying the method of perturbations we obtained two systems of equations, corresponding to terms with or without $\varepsilon$ We used the latter to obtain solutions for $\eta_{0}, u_{0}$, and $x_{0}$, which are the first terms in our approximations of $\eta, u$, and $x_{m}$. Using these first-term solutions in conjunction with the second system of equations (corresponding to $\varepsilon$ ), it should be possible to obtain solutions for $\eta_{1}, u_{1}$, and $x_{1}$, thereby allowing for a closer approximation of the original variables. While the resulting solution will likely be substantially more complicated, it promises even better accuracy. Secondly, as discussed earlier, the existing body of theoretical work on tsunamis to this point focuses rather exclusively on the constant-sloped beach, for which reason we chose to plot our results for that configuration. But while this particular beach configuration represents the most natural starting point for such inquiries, it should be relatively simple to generalize our results to a piecewise-linear beach at the very least. Such results would prove even more helpful in providing engineers with effective predictions regarding the behavior of real-world tsunamis, and how to stop them. 


\section{Acknowledgments}

Thanks to Dr. Vladimir Chugunov of Moscow City University in Moscow, Russia for this formulation of the problem, and for suggesting the method of perturbations for problems of this kind.

This project was funded by the National Science Foundation award: Division of Mathematical Sciences - 1559788.

\section{References}

[1] K. BROWN \& R. CHURCHILL, Fourier series and boundary value problems, McGraw-Hill, New York, NY, 2001.

[2] E. BRYANT, The underrated hazard, Springer, 2008.

[3] G.F. CARRIER \& H.P. GREENSPAN, Water wave of finite amplitude on a sloping beach, J. Fluid Mech., 4.1(1957), pp. 97-109.

[4] G.F. CARRIER, T.T. WU, and H. YEH, Tsunami run-up and draw-down on a plane beach, J. Fluid Mech., 475.1(2003), pp. 79-99.

[5] V. CHUGUNOV, S. FOMIN, and R. SHANKAR, Influence of underwater barriers on the distribution of tsunami waves, J. Geophys. Res. Oceans., 119(2014), pp. 7568-7591.

[6] S. FOMIN \& V. CHUGUNOV, Mathematical modeling of mass transport in complex media, Tohoku University Press, Japan, 2007

[7] J.H. LATTER, Tsunamis of volcanic origin: summary of causes, with particular reference to krakatoa, Bull. Volcanol. 44.3(1883), pp. 467-490.

[8] R. SHANKAR, Y. SHENG, M. GOLBEK, T. HARTLAND, P. GERRODETTE, S. FOMIN, and V. CHUGUNOV, Linear long wave propogation over discontinuous submerged shallow water topography, App. Math. and Comp., 252(2015), pp. 27-44.

[9] J.J. STOKER, Water Waves: The Mathematical Theory With Applications, Interscience Publishers Inc., New York, USA, 1957.

[10] C.E. SYNOLAKIS, The runup of solitary waves, J. Fluid Mech, 185(1987), pp. 523-545.

[11] N.E. VOLTSINGER, K.A. KLEVANNY, and E.N. PELINOVSKY, Shallow Water Theory: Oceanographic Problems and Numerical Methods, Gidro, Leningrad, 1989.

[12] E. ZAUDERER, Partial differential equations of applied mathematics, Wiley, Hoboken, NJ, 2006. 